\title{
Spectrum of neurological disorders in neurology outpatients clinics in urban and rural Sindh, Pakistan: a cross sectional study
}

Safia Awan ${ }^{1}$, Alam Ibrahim Siddiqi ${ }^{2}$, Ahmed Asif ${ }^{3}$, Naveeduddin Ahmed ${ }^{3}$, Hazim Brohi ${ }^{3}$, Sajad Jalbani ${ }^{2}$ and Mohammad Wasay ${ }^{1 *}$ (D)

\begin{abstract}
Background: Neurological disorders are the most common cause of morbidity and mortality in developing countries. Available evidence on urban-rural differences on neurological diseases is scare in such countries. Our study objective was to determine the prevalence of neurological diseases in urban and rural tertiary care hospitals of Sindh, Pakistan.

Methods: This was a cross sectional study conducted in selected urban and rural region of tertiary care hospitals of Sindh, Pakistan. The outpatients medical records of adults (18 years and above) was obtained from January 1st, 2014 to December 31st, 2014.

Results: A total of 10,786 outpatients visit were recorded in this period. Mean age of the participants was $40.6 \pm 15$ years; majority was females 6104 (56.6\%). About three-fourth of the patients were from rural hospital 7828 (72.6\%). Common neurological diseases were headache disorders 3613 (33.4\%), nerve and root lesion 2928 (27.1\%), vascular diseases 1440 (13.3\%), epilepsies 566 (5.2\%), muscle disorders 424 (3.9\%), psychiatric disorders 340 (3.1\%) and CNS infection 303 (2.8\%). Comparison between the urban and rural samples showed that ischaemic stroke $(72.7 \%$ vs. $82 \%)$ and psychiatric disorders $(2.1 \%$ vs. $3.5 \%)$ were more prevalent in rural area as compared to urban setting.
\end{abstract}

Conclusion: Stroke, headache and nerve and root lesion are major causes of neurological disorders in urban and rural settings of Sindh, Pakistan. The policy and planning must be focus on primary care, preventive measures and the promotion of health.

Keywords: Neurological disorders, Stroke, Urban and rural population

\section{Background}

The demographic and epidemiological profile has changed the morbidity and mortality patterns worldwide. Neurological disorders are the fifth leading cause of death globally and are responsible for $5.53 \%$ of total global deaths [1]. Tension-type headache (1505.9 million cases), migraine (958.8 million), medication overuse headache (58.5 million), and Alzheimer's disease including other dementias (46.0 million) were the most prevalent

\footnotetext{
* Correspondence: mohammad.wasay@aku.edu

${ }^{1}$ Department of Medicine and Neurology, Aga Khan University, Karachi, Pakistan

Full list of author information is available at the end of the article
}

neurological disorders. Stroke is the leading cause of deaths accounting for $67.3 \%$ among all neurological disorders followed by Alzheimer's disease and other dementias [2]. Death among young adults due to stroke increased significantly in developing countries and the number of disability-adjusted life years (DALYs) was seven times higher as compared to developed countries $[3,4]$. The World Health Organization estimates that $80 \%$ of all strokes will occur in developing countries [5]. Dementia due to Alzheimer's disease (AD) was more common $60 \%$ than vascular dementia $30 \%$ worldwide [6]. Epilepsy affects approximately 70 million people

(c) The Author(s). 2019 Open Access This article is distributed under the terms of the Creative Commons Attribution 4.0 International License (http://creativecommons.org/licenses/by/4.0/), which permits unrestricted use, distribution, and 
worldwide and $90 \%$ of the people suffering from epilepsy are in developing countries [7].

Stroke is a serious health concern in Pakistan with the annual incidence of $250 / 100,000$ population [8-10]. The incidence of stroke was $21.8 \%$ (with $66.4 \%$ females) according to a study conducted in an urban slum of Karachi, Pakistan [11]. Epilepsy attacked 2.4 million people every year and it crippled 5 million for the rest of their life $[12,13]$. The prevalence of depression and anxiety in Pakistan was 34\% and it is more common among females. Depression is more prevalent in the rural areas $(66 \%$ in women and $25 \%$ in men) than in urban areas $(25 \%$ in women and $10 \%$ in men), [14-16]. Very limited data indicates that the prevalence of Alzheimer's disease (AD) more frequent in elderly population. [17].

Information regarding the urban or rural profiles of neurological disorders obtained from hospitals or community from these areas is important [18]. It reflects the variation between rural and urban areas of the countries; it would also be the bases for policy changes and help to formulate large scale population based intervention studies. Study from India reported that the prevalence rate in urban and rural populations was 2190 and 4070/1,00, 000 respectively [19]. A study conducted in tribal region of Uganda reported that the point prevalence of neurological diseases was 3.3\% [20].

Epidemiological data regarding the neurological conditions is limited in Pakistan. Community based studies to determine the urban-rural differences of neurological diseases are very difficult to perform due to lack of resources, and because the number of neurologist is limited.

Previous work in Pakistan is based on specific diseases conditions and most were conducted in urban centers. The need would be to show the spectrum of neurological diseases and compare how their profile differs in urban and rural patients presenting to outpatient settings.

Therefore, our study objective was to determine the prevalence of neurological diseases in urban and rural tertiary care hospitals of Sindh, Pakistan. This dataset of different neurological diagnoses in urban and rural tertiary care hospitals serves as a starting point to inform policy and open up venue for further research. Sind is second largest province of Pakistan with a population of 47.89 Million.

\section{Methods}

\section{Study design}

This is a cross-sectional study conducted using outpatient medical records. Data on all adult (18 years and above) patients presenting to the selected outpatient clinic was obtained from January 2014 to December 2014.

\section{Study setting}

The study was conducted in two tertiary care hospitals of the Sindh province of Pakistan representing urban and rural populations. Liaquat National Hospital \& Medical College is a major semi-private hospital located in Karachi city that has a population of 14.9 million as recorded in the 2017 Census of Pakistan. Shaheed Mohtarma Benazir Bhutto Medical University (SMBBMU) is a public university of medicine, Nursing Health and medical sciences located in rural district of Larkana (population 1.5 Million).

\section{Data collection}

The tool of the study has been reported previously and here we provide a brief summary [13]. A structured questionnaire was used to collect data on patient's age, gender, place and type of residence (rural/urban), referral and patient's evaluation status and diagnosis. The diagnosis was made by neurologist of the outpatient facility of selected hospitals. The neurological conditions were grouped according to International Classification of Disease [21] as follows: 1) Vascular diseases which include all type of stroke, moya moya disease, unruptured saccular aneurysm and arteriovenous malformation. 2) Cerebellar ataxia and hereditary spastic paraplegias include motor neuron diseases and sleep disorders. 3) Headache disorders 4) Acquired metabolic and toxic disorders which include all types of encephalopathy, psychosis, ataxia and delirium tremens. 5) CNS infections which include all type of meningitis, mucormycosis and herpes zoster. 6) CNS neoplasms, 7) Nerve and root lesion which includes neuritis, radiculitis, neuropathy, Guillain Barre Syndrome (GBS) and cranial nerve disorders. 8) Psychiatric disorders which include depression, conversion and bipolar disorder, anxiety neurosis, obsessive compulsive disorders, psychosis, and drug abuse. 9) Demyelinating disease 10) dementias 11) myopathies/muscle disorders, 12) developmental disorders 13) movement disorders, 14) epilepsies, 15) spinal disorders, and 16) neurometabolic/nutritional disorders of the nervous system.

\section{Statistical analysis}

Descriptive analysis for demographic variables was performed; results are reported as numbers with percentages for quantitative variables. Continuous variables with normal and non-normal distributions are reported as mean (SD) and median [inter-quartile range (IQR)], respectively. Comparison between urban and rural population with neurological diseases were performed using Pearson Chi-square test and Continuous variables were compared using Student t test. All $p$-values were two sided and $<0.05$ was considered as statistically significant. 
Table 1 Descriptive characteristics of study population $(n=10,786)$

\begin{tabular}{ll}
\hline & $\mathrm{n}(\%)$ \\
\hline Age, in years & $40.6 \pm 15.0$ \\
Gender & \\
Male & $4682(43.4)$ \\
Female & $6104(56.6)$ \\
Residence & \\
Rural & $7828(72.6)$ \\
Urban & $2958(27.4)$ \\
Status ( $n=10,776)$ & \\
New & $6164(57.2)$ \\
Follow-up & $4612(42.8)$ \\
Referral ( $n=10,783)$ & \\
Self & $2340(21.7)$ \\
Physician & $6166(57.2)$ \\
Family/friends & $2277(21.1)$ \\
Vascular diseases ( $n=1440)$ & \\
Stroke & $1431 / 1440(99.3 \%)$ \\
Ischaemic Stroke Acute/old & $1141(79.7)$ \\
Transient Ischaemic attack & $37(2.5)$ \\
Haemorrhagic stroke (parenchymal bleed) & $253(17.6)$ \\
\hline
\end{tabular}

Cerebellar Ataxias and Herediatory spactic paraplegias $(n=42)$

Motor Neuron diseases

$28(66.6)$

Cerebellar ataxia

4 (9.5)

Cerebellar Degeneration

$9(21.4)$

Headache Disorders $(n=3613)$

Migraine

957 (26.6)

Tension type headache

$2631(73)$

Occipital Headache

$14(0.4)$

Sleep disorders $(n=36)$

Narcolepsy

$4(11.1)$

Obstructive sleep apnoea

$5(13.8)$

Restless leg syndrome

Acquire Metabolic and Toxic Disorders $(n=8)$

CNS Infections ( $n=303)$

Bactrial meningitis

$156(51.4)$

Tuberculous meningitis / Tuberculoma

$47(15.5)$

Brain abscess

$11(3.6)$

Herpes zoster ophthalmicus/shingles

$9(2.9)$

Tuberculoma

$62(20.4)$

CNS Neoplasms $(n=35)$

Meningioma

$10(28.5)$

Glioma

$7(0.2)$

Astocytoma grade I-IV (GBM)

$4(11.4)$

Pituitary adenoma/ Craniopharyngioma
Table 1 Descriptive characteristics of study population $(n=10,786)$ (Continued)

\begin{tabular}{|c|c|}
\hline & $\mathrm{n}(\%)$ \\
\hline Metastatic brain tumor & $8(22.8)$ \\
\hline \multicolumn{2}{|l|}{ Nerve and root lesions $(n=2928)$} \\
\hline Guillain Barre Syndrome (GBS) & $35(1.1)$ \\
\hline CIDP & $57(1.9)$ \\
\hline Diabetic polyneuropathy & $263(8.9)$ \\
\hline Cervical radiculopathy & $761(25.9)$ \\
\hline Lumber radiculopathy & $1589(54.2)$ \\
\hline \multicolumn{2}{|l|}{ Myopathies/Muscle Disorders $(n=424)$} \\
\hline Polymyositis & $30(7.0)$ \\
\hline Myasthenia gravis & $15(3.5)$ \\
\hline Fibromyalgia & $12(2.8)$ \\
\hline Musculoskeltal pain & $315(74.2)$ \\
\hline Hypokalemic periodic paralysis & $12(2.8)$ \\
\hline \multicolumn{2}{|l|}{ Dementias $(n=194)$} \\
\hline Alzheimer's diseases & $116(59.7)$ \\
\hline Vascular dementia & $60(30.9)$ \\
\hline \multicolumn{2}{|l|}{ Psychiatric Disorders $(n=340)$} \\
\hline Depression & $171(50.3)$ \\
\hline Bipolar disorder & $94(27.6)$ \\
\hline Anxiety neurosis & $10(2.9)$ \\
\hline Obsessive compulsive disorders & $15(4.4)$ \\
\hline Conversion disorders & $32(9.4)$ \\
\hline \multicolumn{2}{|l|}{ Demyellinating Diseases $(n=59)$} \\
\hline Multiple sclerosis & $29(49.1)$ \\
\hline Acute disseminated encephalomyelitis (ADEM) & $8(13.5)$ \\
\hline Cerebellar Ataxias \& Hereditary spastic paraplegias & $6(10.1)$ \\
\hline Motor neurone diseases & $9(15.2)$ \\
\hline \multicolumn{2}{|l|}{ Movement Disorders $(n=231)$} \\
\hline Parkinson's diseases & $154(66.6)$ \\
\hline Chorea & $16(6.9)$ \\
\hline Tics & $10(4.3)$ \\
\hline Dystonias & $14(6.0)$ \\
\hline Cerebral palsy (CP) & $13(5.6)$ \\
\hline Hemifacial spasm (HFS) & $19(8.2)$ \\
\hline \multicolumn{2}{|l|}{ Epilepsies $(n=566)$} \\
\hline Focal Epilepsy & $29(5.1)$ \\
\hline Generalized Epilepsy & $529(93.4)$ \\
\hline \multicolumn{2}{|l|}{ Spinal Disorders $(n=156)$} \\
\hline Myelopathy (Spinal cord diseases) & $124(79.4)$ \\
\hline Syringomyelia & $14(8.9)$ \\
\hline
\end{tabular}


Data entry and data analysis was done by using SPSS 19.0 (SPSS Inc., Chicago: IL, USA).

\section{Results}

A total of 10,786 outpatient visits were recorded across both sites during the study period. Mean age of the participants was $40.6 \pm 15$ years with a median age [IQR] of 40[29-50] years; 4682 (43.4\%) males, 6104 (56.6\%) females. Majority of the participants were from rural hospital 7828 (72.6\%). Common neurological diseases were headache disorders 3613 (33.4\%), nerve and root lesion 2928 (27.1\%), vascular diseases 1440 (13.3\%), epilepsies 566 (5.2\%), muscle disorders 424 (3.9\%), psychiatric

Table 2 Comparison of neurological disorders in urban and rural areas $(n=10,786)$

\begin{tabular}{|c|c|c|c|}
\hline & Rural; $n=7828$ n (\%) & Urban; $n=2958$ n (\%) & $p$ value \\
\hline Age in years; mean $\pm S D$ & $40.8 \pm 15.2$ & $40.2 \pm 14.6$ & 0.09 \\
\hline \multicolumn{4}{|l|}{ Gender } \\
\hline Male & $3635(46.4)$ & $1047(35.4)$ & \multirow[t]{2}{*}{$<0.001$} \\
\hline Female & $4193(53.6)$ & $1911(64.6)$ & \\
\hline \multicolumn{4}{|l|}{ Stroke $(n=1431)$} \\
\hline Ischaemic stroke acute/old & $891(82)$ & $250(72.7)$ & \multirow[t]{3}{*}{$<0.001$} \\
\hline Transient Ischaemic attack & $19(1.7)$ & $18(5.2)$ & \\
\hline Haemorrhagic stroke & $177(16.3)$ & $76(22.1)$ & \\
\hline \multicolumn{4}{|l|}{ Headache Disorders ( $n=3613$ ) } \\
\hline Migraine & $623(24.6)$ & $334(31.2)$ & \multirow[t]{3}{*}{$<0.001$} \\
\hline Tension type Headache & $1900(75)$ & $731(68.4)$ & \\
\hline Occipital headache & $10(0.4)$ & $4(0.4)$ & \\
\hline CNS infection $(n=303)$ & $210(2.7)$ & $93(3.1)$ & 0.19 \\
\hline Meningitis; $n=210$ & $144(68.6)$ & $66(71)$ & 0.67 \\
\hline \multicolumn{4}{|l|}{ Meningitis } \\
\hline Bacterial Meningitis & $107(74.3)$ & $49(74.2)$ & \multirow[t]{4}{*}{0.47} \\
\hline Tuberculous/Tuberculoma & $34(23.6)$ & $13(19.7)$ & \\
\hline Fungal meningitis & $1(0.7)$ & $1(1.5)$ & \\
\hline Viral meningitis & $2(1.4)$ & $3(4.5)$ & \\
\hline Nerve and root lesions; $n=2928$ & $2084(26.6)$ & $844(28.5)$ & 0.04 \\
\hline Guillain Barre syndrome (AIDP) & $26(1.2)$ & $9(1.1)$ & 0.68 \\
\hline CIDP & $45(2.2)$ & $12(1.4)$ & 0.19 \\
\hline Diabetic polyneuropathy & $161(7.7)$ & $102(12.1)$ & $<0.001$ \\
\hline Cervical radiculopathy & $546(26.2)$ & $215(25.5)$ & 0.68 \\
\hline Lumber radiculopathy & $1146(55)$ & $443(52.5)$ & 0.21 \\
\hline Psychiatric disorders; $n=340$ & $277(3.5)$ & $63(2.1)$ & $<0.001$ \\
\hline Depression; $n=171$ & $125(45.1)$ & $46(73)$ & $<0.001$ \\
\hline Alzheimer's diseases; $n=116$ & $86(57.7)$ & $30(66.7)$ & 0.28 \\
\hline Musculoskeletal pain; $n=315$ & $225(73.3)$ & $90(76.3)$ & 0.52 \\
\hline Movement disorders; $n=221$ & $167(2.1)$ & $54(1.8)$ & 0.30 \\
\hline Parkinsons; $n=150$ & $117(70.1)$ & $33(61.1)$ & 0.22 \\
\hline Epilepsy; $n=566$ & $408(5.2)$ & $158(5.3)$ & 0.78 \\
\hline \multicolumn{4}{|l|}{ Epilepsy type } \\
\hline Focal Epilepsy & $21(5.1)$ & $8(5.1)$ & \multirow[t]{2}{*}{0.06} \\
\hline Generalized Epilepsy & $361(88.5)$ & $148(93.7)$ & \\
\hline Myelopathy; $n=124$ & $96(80)$ & $28(77.8)$ & 0.77 \\
\hline Spinal disorders; $n=156$ & $120(1.5)$ & $36(1.2)$ & 0.24 \\
\hline
\end{tabular}


disorders 340 (3.1\%) and CNS infection 303 (2.8\%); Table 1.

Comparison between the rural and urban samples showed that Ischaemic stroke $(82 \%$ vs. $72.7 \%)$ and psychiatric disorders $(3.5 \%$ vs. $2.1 \%)$ were more prevalent in rural area as compare to urban population. However, headache disorders $(32.1 \%$ vs. $35.3 \%)$, nerve and root lesions $(28.5 \%$ vs. $26.6 \%)$ diabetes polyneuropathy $(12.1 \%$ vs. $7.7 \%$ ) and depression ( $73 \%$ vs. $45.1 \%)$ were comparatively higher in urban area as compare to rural area and this was the significant difference between the two populations Table 2.

Among the 7828 rural participants, the most prevalent neurological diagnoses were headache 1910 (24.4\%), lumber radiculopathy 1146(14.6\%), stroke 1087(13.9\%), migraine $623(8.0 \%)$, cervical radiculopathy $546(7.0 \%)$ and epilepsy 408 (5.2\%).

Among the 2958 urban participants, the most prevalent diagnoses were headache 735 (24.8\%), lumber radiculopathy $443(15 \%)$, stroke $344(11.6 \%)$ and epilepsy $158(5.3 \%)$.

A stratification of participants by gender is represented in Table 3. Headache disorders were the most common type prevalent among females.

\section{Discussion}

This is the first study to compare the neurological disorders among urban and rural population. The demographic characteristics of the study indicate that neurological disorders were more prevalent among women (56.6\%) and in 30 to 50 years of age group. Previous study from Karachi reported the age group between 46 and 65 years which represented $34 \%$ of all out-patient neurological visits [13]. WHO, estimates show that $20 \%$ of adults aged 60 and over suffer from a mental or neurological disorder [22, 23]. A community based study from India reported the higher prevalence of neurological disorder among women as compared to men [19].

The first three prevalent disorders for both urban and rural areas are stroke, headache disorders and nerve and root lesions disorders. Almost similar trend was reported in previous studies [20, 24, 25]. A study from Karachi shows that the leading diagnoses were stroke $98.2 \%$, Parkinson's $67.7 \%$ and depression $77.5 \%$ [13].
A $72.5 \%(7828 / 10786)$ of participants was presented in the rural hospital as compared to $27.5 \%$ from urban center in our study. Only one government run tertiary care hospital is available to cater this population. The Basic Health Units and Rural dispensaries set up by the provincial and district governments in some villages, mostly have no doctors. Additionally, attracting and retaining well-qualified medical personnel is more challenging in rural environments.

Stroke is more prevalent in urban area but ischaemic stroke is more prevalent in rural area as compared to urban area. Most regions will see an increasing trend of stroke with the most notable rises in South Asia [26]. Similarly, stroke prevalence was higher in rural areas of India than in urban areas [27]. On the contrary, the incidence is higher in urban areas than in rural areas of Indonesia [28] and Thailand [29].

The overall prevalence of headache disorders was 33\% and this is more prevalent in urban areas as compared to rural areas. This is slightly higher as compared to earlier study from India [30] but prevalence is higher among those from rural areas than urban [30]. Higher proportion of females is an almost consistent finding in many other studies [31].

The rate of neurologists in high income countries is 402 neurologists per 100,000 inhabitants while in low income countries it is only 4.5 per 100,000 inhabitants [32]. The number of beds for neurology patients is significantly lowered in low-income versus high-income regions in public hospitals $(0.19 / 100,000$ versus $11.73 / 100,000)$. In Pakistan, the rate is 1 neurologist per 1.5 million inhabitants [13, 33].

In Pakistan, most of the population doesn't have access to neurology specialist in secondary and district level hospitals. The huge burden of patients and limited number of resources available in government hospitals made the situation worse for physicians. There is need to restructure the neurology training program. There is urgent need to develop a share model of teaching neurology program for general physicians and undergraduate level. Moreover, focus must be given to develop proper infrastructure and diagnostic facilities in the hospitals.

To our knowledge, this is the first study comparing rural and urban settings from Pakistan, Almost, $60.3 \%$ population in Pakistan lived in rural areas. Secondly, our study patients were evaluated and examination by neurologist.

Table 3 neurological disorders and sex distribution among rural and urban population

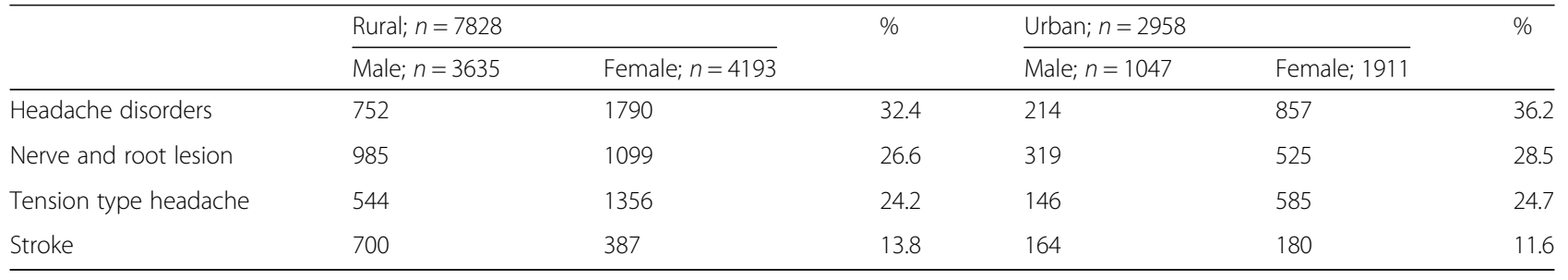


This study is not without limitations; this study was a hospital based and the fact that prevalence or incidence is better calculated by community-based prospective studies.

\section{Conclusion}

Our study showed significant differences on different type of neurological disorders between the urban and rural populations. A headache disorder was the most frequent neurological condition in the rural population while stroke was more prevalent in urban population.

\section{Additional file}

Additional file 1: Description of data: demographic and clinical characteristics. (DOCX 96 kb)

\section{Abbreviations}

AD: Alzheimer's disease; AIDP: Guillain Barre Syndrome; DALYs: Disabilityadjusted life years; NCDs: Neurological disorders

\section{Acknowledgements}

We would like to thank data collector teams for their support towards the study.

\section{Authors' contributions}

MW developed the idea, protocol writing and manuscript review. SA designs the study, wrote the manuscript and performed statistical analysis. AIS reviewed and approved the drafts of the manuscript. AA helped drafting of manuscript. NA helped data interpretation, manuscript writing and manuscript reviewing. $\mathrm{HB}$ and $\mathrm{SJ}$ wrote the draft and review the final manuscript. All authors read and approved the final manuscript.

\section{Funding}

None

\section{Availability of data and materials}

The datasets generated and/or analysed during the current study are available from the corresponding author on reasonable request (Additional file 1).

\section{Ethics approval and consent to participate}

The study was approved by Aga Khan University (AKU) Ethical Review Committee (ERC). We used only recorded medical data; the ERC at our center approved the study and waived the requirement for informed consent.

\section{Consent for publication}

Not applicable

\section{Competing interests}

The authors declare that they have no competing interests.

\section{Author details}

'Department of Medicine and Neurology, Aga Khan University, Karachi, Pakistan. ${ }^{2}$ Department of Neurology, Shaheed Mohtarma Benazir Bhutto Medical University, Larkana, Pakistan. ${ }^{3}$ Department of Neurology, Liaquat National Hospital, Karachi, Pakistan.

Received: 1 October 2018 Accepted: 7 August 2019

Published online: 13 August 2019

\section{References}

1. HME. GBD Data Visualizations. 2017. cited; Available from: https://vizhub. healthdata.org/gbd-compare/.

2. Feigin VL, et al. Global, regional, and national burden of neurological disorders during 1990-2015: a systematic analysis for the Global Burden of Disease Study 2015. Lancet Neurol. 2017;16(11):877-97.
3. Feigin $\mathrm{VL}$, et al. Global burden of stroke and risk factors in 188 countries, during 1990-2013: a systematic analysis for the Global Burden of Disease Study 2013. Lancet Neurol. 2016;15(9):913-24.

4. Mathers CD, Lopez AD, Murray CJL. The burden of disease and mortality by condition: data, methods and results for 2001. In: Global burden of disease and risk factors, vol. 45; 2006. p. 88

5. Mathers CD, Loncar D. Updated projections of global mortality and burden of disease, 2002-2030: data sources, methods and results. Geneva: World Health Organization; 2005

6. Kalaria RN, et al. Alzheimer's disease and vascular dementia in developing countries: prevalence, management, and risk factors. Lancet Neurol. 2008;7(9):812-26.

7. Singh A, Trevick S. The epidemiology of global epilepsy. Neurol Clin. 2016:34(4):837-47.

8. Khealani BA, Hameed B, Mapari UU. Stroke in Pakistan. J Pak Med Assoc. 2008:58(7):400.

9. Hashmi M, Khan M, Wasay M. Growing burden of stroke in Pakistan: a review of progress and limitations. Int J Stroke. 2013;8(7):575-81.

10. Wasay M, Khatri IA, Kaul S. Stroke in south Asian countries. Nat Rev Neurol. 2014;10(3):135.

11. Kamal AK, et al. The burden of stroke and transient ischemic attack in Pakistan: a community-based prevalence study. BMC Neurol. 2009;9(1):58.

12. Hussain $\mathrm{G}$, et al. Epidemiological data of neurological disorders in Pakistan and neighboring countries: a review. PJNS. 2017;12(4):52-70.

13. Awan S, et al. Pattern of neurological diseases in adult outpatient neurology clinics in tertiary care hospital. BMC Res Notes. 2017;10(1):545

14. Zahid N, et al. Depression and diabetes in a rural community in Pakistan. Diabetes Res Clin Pract. 2008;79(1):124-7.

15. Mumford DB, et al. Stress and psychiatric disorder in rural Punjab: a community survey. Br J Psychiatry. 1997;170(5):473-8.

16. Husain N, Creed F, Tomenson B. Depression and social stress in Pakistan. Psychol Med. 2000;30(2):395-402.

17. Usman S, et al. Severity and risk factors of depression in Alzheimer's disease. J Coll Physicians Surg Pak. 2010;20(5):327-30.

18. Siddiqi OK, et al. The spectrum of neurological disorders in a Zambian tertiary care hospital. J Neurol Sci. 2010;290(1):1-5.

19. Gourie-Devi M, et al. Prevalence of neurological disorders in Bangalore, India: a community-based study with a comparison between urban and rural areas. Neuroepidemiology. 2004;23(6):261-8.

20. Kaddumukasa $M$, et al. Prevalence and incidence of neurological disorders among adult Ugandans in rural and urban Mukono district; a cross-sectional study. BMC Neurol. 2016;16(1):227.

21. World Health, O. International statistical classification of diseases and related health problems, 10th revision (ICD-10), vol. 2010. Geneva: WHO; 2010.

22. Dong X. Elder abuse: research, practice, and health policy. The 2012 GSA Maxwell Pollack Award Lecture. Gerontol. 2014;54(2):153-62 [cited 2017 Feb 07].

23. Callixte K-T, et al. The pattern of neurological diseases in elderly people in outpatient consultations in sub-Saharan Africa. BMC Res Notes. 2015;8(1):159.

24. Uddin MS, et al. Spectrum of disease and prescription pattern for outpatients with neurological disorders: an empirical pilot study in Bangladesh. Ann Neurosci. 2018;25(1):25-37.

25. Habeych ME, GarcÃa-Habeych JM, Castilla-Puentes RC. Different neurological conditions between urban and rural samples from Central Colombia. J Neurol Sci. 2012;320(1):56-60.

26. Lloyd-Sherlock P. Stroke in developing countries: epidemiology, impact and policy implications. Dev Policy Rev. 2010;28(6):693-709.

27. Pandian JD, Sudhan P. Stroke epidemiology and stroke care services in India. J Stroke. 2013;15(3):128.

28. Kusuima Y, et al. Burden of stroke in Indonesia. I Int J Stroke. 2009;4(5):379-80.

29. Suwanwela NC. Stroke epidemiology in Thailand. J Stroke. 2014;16(1):1.

30. Kulkarni $G B$, et al. Headache disorders and public ill-health in India: prevalence estimates in Karnataka state. J Headache Pain. 2015:16(1):67.

31. Bolay $\mathrm{H}$, et al. Gender influences headache characteristics with increasing age in migraine patients. Cephalalgia. 2015;35(9):792-800.

32. Neurology, W.F.o. WCN 2015 - High global burden of neurological diseases recognized. 2015. cited; Available from: https://www.wfneurology.org/2 015-10-31-wfn-wcn-high-global-burden.

33. Hayat M, et al. Future of neurology in Pakistan. J Pak Med Assoc. 2003;53(12):576-9.

\section{Publisher's Note}

Springer Nature remains neutral with regard to jurisdictional claims in published maps and institutional affiliations. 